\title{
Correction to: The End of Epicurean Infinity: Critical Reflections on the Epicurean Infinite Universe
}

Frederik A. Bakker

\section{Correction to:}

Chapter 3 in: F. A. Bakker et al. (eds.), Space, Imagination and the Cosmos from Antiquity to the Early Modern Period, Studies in History and Philosophy of Science 48, https://doi.org/10.1007/978-3-030-02765-0_3

Owing to an oversight on the part of Springer, Chapter 3 was initially published as a regular chapter. However, this is an Open Access chapter. 